\title{
Violência conjugal e família de origem: perfil discriminante de parceiros que cometem e não cometem infidelidade
}

\author{
Patrícia Manozzo Colossi ${ }^{1}$ \\ Denise Falcke ${ }^{2}$ \\ ${ }^{\prime}$ Faculdades Integradas de Taquara, Taquara, RS, Brasil, \\ Universidade do Vale do Rio dos Sinos, São Leopoldo, RS, Brasil \\ ${ }^{2}$ Universidade do Vale do Rio dos Sinos, São Leopoldo, RS, Brasil
}

\begin{abstract}
Resumo
Este estudo identificou como os participantes que cometeram e os que não cometeram infidelidade se diferenciam quanto à violência conjugal e experiências na família de origem. Foram pesquisados 384 mulheres e 216 homens (n=600), maiores de idade, em relacionamento de namoro, casamento ou união estável há pelo menos seis meses, recrutados por mídias sociais e acessados por plataforma digital. Os instrumentos foram: questionário de dados sociodemográficos, Revised Conflict Tactics Scale, questionário de experiências familiares e escalas de infidelidade (percepções-PDIS, atitudes-EAI e propensão-EPI). Os resultados revelaram a propensão e as atitudes relacionadas à infidelidade como dimensões discriminantes entre os grupos. As experiências de negligência física, abuso sexual, ajustamento psicológico materno/paterno, abuso físico materno e aliança parental na família de origem revelaram maior poder discriminante; e a agressão psicológica foi a expressão violenta que apresentou maior discriminação entre os grupos. Os resultados indicam a relevância de enfocar os aspectos associados à violência como forma de prevenção, nos âmbitos primário, secundário e terciário.
\end{abstract}

Palavras-chave: Infidelidade; Família de origem; Violência conjugal; Perfil discriminante.

\section{Intimate partner violence and family of origin: discriminant profile of partners who commit and do not commit infidelity}

\begin{abstract}
This study identified how participants who committed and those who did not commit infidelity differ in terms of marital violence and experiences in the family of origin. Was researched 384 women and 216 men $(n=600)$, of legal age, dating, marriage or stable union were surveyed for at least six months, recruited by social media and accessed by digital platform. The instruments used were: sociodemographic data questionnaire, Revised Scale of Conflict Tactics, family experiences questionnaire and infidelity scales (perceptions- PDIS, attitudes-EAI, propensity- EPI). The results revealed propensity and attitudes related to infidelity as discriminant dimensions between groups. The experiences of physical neglect, sexual abuse, maternal/paternal psychological adjustment, maternal physical abuse and parental alliance in the family of origin revealed greater discriminant power and psychological aggression was the violent expression that presented greater discrimination between the groups. The results point out the relevance of focusing the aspects associated with violence as a form of prevention, in the primary, secondary and tertiary environments.
\end{abstract}

Keywords: Infidelity; Family of origin; Intimate partner violence; Discriminant profile.

\section{Violencia entre pareja y familia de origen: perfil discriminante de los compañeros que cometen y no cometen infidelidad}

\section{Resumen}

Este estudio identificó como participantes que han cometido y los que cometen infidelidad diferentes en cuanto a la violencia doméstica y experiencias en la familia de origen. 384 mujeres fueron encuestadas, 216 hombres $(n=600)$, mayor de edad, en las citas, matrimonio o unión estable durante al menos seis meses, reclutados por medios sociales y accedidos por plataforma digital. Los instrumentos utilizados fueron: cuestionario sociodemográfico, Escala de tácticas de conflicto revisadas, experiencias familiares cuestionario y escalas de infidelidad (PDIS percepciones, actitudes y propensión a IAC EPI). Los resultados mostraron parcialidad y actitudes a la infidelidad como dimensiones discriminantes entre los grupos. Las experiencias de abandono físico, el abuso sexual, el ajuste psicológico materno / paterno, el abuso físico materna y la alianza de los padres en la familia de origen mostraron un mayor poder discriminante y la agresión psicológica fue la expresión violenta que mostró una mayor discriminación entre los grupos. Los resultados muestran la importancia de centrarse en los aspectos asociados a la violencia como medio de prevención en entornos primarios, secundarios y terciarios.

Palabras clave: Infidelidad; Familia de origen; La violencia de pareja; Perfil discriminante. 


\section{Introdução}

Os fenômenos associados aos relacionamentos íntimos têm sido investigados há muito tempo na tentativa de compreender os aspectos que contribuem para que as relações amorosas sejam duradouras ou não (Féres-Carneiro, 1998, Féres-Carneiro \& Neto, 2010; Jablonski, 1991; Rizzon, Mosmann, \& Wagner, 2013). Diferentes aspectos individuais e relacionais contribuem para o estabelecimento de uma dinâmica conjugal específica, relacionados a um contrato conjugal único para cada casal (Costa \& Cenci, 2014; Silva, Rocha, Bobato, Becker, \& Lorenzetti, 2015).

Aspectos como uma expectativa frágil frente ao casamento, valorização da independência e individualismo, mudança do papel da mulher na conjugalidade, família e sociedade, influências da família de origem e infidelidade conjugal são apontados como preditores de separações e/ou divórcios conjugais (Zordan, 2010). No que tange à infidelidade, chama a atenção a recorrência e complexidade dos casos evidenciados na clínica conjugal e familiar (Prado, 2012). Ainda que seja uma realidade observada em diferentes épocas e contextos sociais, as taxas de prevalência têm aumentado de modo relevante nos últimos anos (Pasini, 2010). Das pessoas pesquisadas por Donovan \& Emmers-Sommer (2012), 22\% admitiram ter sido infiéis com o parceiro atual, enquanto $50 \%$ dos respondentes revelaram infidelidade em algum relacionamento ao longo da vida. Os dados, contudo, não são concordantes, já que outros estudos referem que entre $26 \%$ e $70 \%$ das mulheres e $33 \%$ e $75 \%$ dos homens são infiéis em seus relacionamentos amorosos (Eaves \& RobertsonSmith, 2007; Jeanfreau, Jurich, \& Mong, 2013). A discrepância dos referidos índices deve-se ao método e delineamento empregados, tipo de amostra e contexto social, bem como à definição de infidelidade atribuída pelos pesquisadores. Assim, a prevalência de infidelidade pode ser maior, considerando a dificuldade de estabelecimento de consenso em relação à definição de comportamentos infiéis (Andrade, Mello, \& Dias, 2009; Mattingly, Wilson, Clark, Bequette, \& Weidler, 2010; Rokach \& Philibert-Lignières, 2015). Soma-se a isso, o fato de muitos casos permanecerem encobertos, sem que as pessoas desejem revelá-los.

Destaca-se contudo, que ao referir o termo infidelidade, não se consideram os casos de poliamor, as relações amorosas abertas ou situações de swing, já que o que difere o contexto de infidelidade das referidas situações amorosas é, justamente, a quebra de um contrato estabelecido entre os cônjuges. Se os parceiros não estabelecem um acordo de exclusividade amorosa e/ou sexual, não são enquadrados, assim, como relacionamentos infiéis (Pittman, 1994). O poliamor, por exemplo, caracteriza-se por um tipo de relacionamento em que são possíveis mais de uma relação afetivo-amorosa simultânea com o consentimento dos parceiros envolvidos (França, 2015; Pilão, 2012), divergindo dos casos de infidelidade em que não se verifica o consentimento do parceiro para o estabelecimento de uma outra relação.

Um estudo realizado ainda na década de 1990, apontava a representação negativa que o fenômeno da infidelidade assumia para a maioria das pessoas, comumente relacionada a uma falha de caráter (Atwood $\&$ Seifer, 1997), revelando um aspecto paradoxal e de difícil compreensão por tratar-se de um constructo complexo e conceitualmente frágil (Scheinkman, 2008). Em uma percepção com maior carga moral, Pittman (1994) define a infidelidade como traição, por considerá-la o rompimento de um acordo, podendo causar diferentes efeitos psicopatológicos incluindo desilusão em relação ao parceiro, profunda tristeza e baixa autoestima (Horta \& Daspett, 2010).

Em sentido distinto, desde a década passada, estudos sobre o tema apontavam a infidelidade como um fenômeno tão recorrente quanto incompreendido nas relações amorosas (Atkins, Baucom, \& Jacobson, 2001). Outros autores definem o fenômeno como infidelidade, em detrimento do termo traição, por contemplar, desde a definição, menor carga moral e referindo um comportamento possível em uma relação conjugal (Viegas \& Moreira, 2013) até mesmo sem causar estranheza aos envolvidos quando consideram que a relação já não vai bem (Mendonça, 2009). A partir de uma compreensão sistêmica, que sustenta o presente estudo, a infidelidade pode ser compreendida como um aspecto da dinâmica conjugal não significando, necessariamente uma falha individual (Mendonça, 2009).

Costa e Cenci (2014) referem que "a infidelidade não se encerra em si mesma” (p.20), destacando a necessidade de compreender a relação conjugal de modo amplo, a partir dos aspectos que influenciam a forma como os parceiros se relacionam. Ademais, cabe considerar que os fatores que constituem a conjugalidade são idiossincráticos e contemplam não somente a individualidade dos parceiros, mas também os aspectos relacionais, as condições socioculturais, as vivências em suas famílias de origem e demais relações que os parceiros se envolvem ao longo da vida.

A influência das experiências vivenciadas no núcleo familiar e sua repercussão no desenvolvimento e estabelecimento de relações do sujeito ao longo da vida são foco de diferentes estudos, ao redor do mundo (Colossi, Marasca, \& Falcke, 2015; Gover, Park, 
Tomsich, \& Jennings, 2011; Kerley, Xu, Sirisunyaluck, \& Alley, 2010; Pedro, 2013). Essas vivências na família de origem podem ser recordadas positivas ou negativas (Melchert, 1998) e assumem um peso diferente na vida do sujeito; conforme a qualidade, frequência, intensidade e carga emocional com que se apresentam. Podem, assim, contribuir em alguma medida para a dinâmica relacional estabelecida pelo casal.

Estudos têm buscado identificar o modo como lembranças dos filhos acerca da relação de seus pais podem estar associadas à forma como irão estabelecer suas relações amorosas na vida adulta, evidenciando o impacto das experiências na família de origem na constituição do sujeito (Braga \& Dell'Aglio, 2012; Marasca, Colossi, \& Falcke, 2013; Moré \& Krenkel, 2014; Ziviani, Féres-Carneiro, \& Magalhães, 2011, 2012). Associado a outros aspectos, é possível que, se a interação do casal parental for percebida pelos filhos como positiva, suas possiblidades relacionais sejam funcionais. Ao contrário, se os filhos perceberem a interação dos pais como negativa, é possível que haja um prejuízo em seu processo de estruturação psíquica e relacional, contribuindo para o estabelecimento de relações conflitivas e disfuncionais na vida adulta (Colossi, Marasca, \& Falcke, 2015; Scorsolini-Comin, Fontaine, \& Santos, 2015; Marasca, Colossi, \& Falcke, 2013).

Estudos revelam que as experiências negativas na família de origem, em especial as vivências de violência, como vítima ou como testemunha, contribuem de modo relevante, para o envolvimento dos filhos em contextos relacionais violentos na vida adulta (Alves, Pinto, Silveira, Oliveira, \& Melo, 2012; Pinheiro, Crepaldi, \& Cruz, 2012). Os pais, por geralmente se constituírem a primeira referência de sujeito a qual os filhos têm contato, assumem destacada relevância como modelo de identificação aprendido. Com isso, as características relacionais do casal parental assumem um papel significativo, já que ensinam aos filhos o que, afinal, é ser um casal. Neste sentido, o contexto conjugal violento acaba por ensinar aos filhos que a violência é uma alternativa viável de relacionamento interpessoal (Marasca, Colossi, \& Falcke, 2013), naturalizando-a como algo esperado em qualquer relacionamento. Com isso, os filhos que crescem em um contexto familiar com baixos índices de aliança parental podem repetir os padrões negativos vivenciados, levando para seus relacionamentos futuros o modelo relacional violento (Falcke, 2006; Marasca, Colossi, \& Falcke, 2013).

Além disso, a saúde mental dos pais, definida neste estudo como ajustamento psicológico (Melchert, 1998), contribui de forma relevante para que os filhos cresçam em um contexto familiar equilibrado e estável do ponto de vista emocional. A literatura refere que o ajustamento psicológico dos pais tem potencial relevante para repercutir na qualidade da relação conjugal dos filhos (Ha, Overbeek, Vermulst, \& Engels, 2009; Snyder \& Lopez, 2009; Wong, McElwain, \& Halberstadt, 2009).

Contudo, não se trata de considerar uma compreensão determinista, em uma perspectiva linear de causa e efeito, já que outros aspectos contribuem para o envolvimento afetivo (Falcke, Wagner \& Mosmann, 2008). O que se destaca é que a percepção que os filhos têm da aliança parental contribui de alguma forma para a compreensão que eles estabelecem acerca de um relacionamento amoroso (Riggio \& Weiser, 2008). Assim, considerando a naturalização das experiências na família de origem, é possível que os filhos acabem perpetuando padrões relacionais presentes na conjugalidade dos pais.

A partir das diferentes interações que envolvem a violência entre parceiros íntimos, a família se mostra, geralmente, como o principal locus de expressão, sendo possível identificar a violência como um fenômeno interacional. Deste modo, a violência não pode ser compreendida como uma construção individual, mas como uma trama relacional em que "todos integrantes estão envolvidos e se afetando mutuamente, sendo que sua expressão dependerá do contexto histórico, social e geracional em que violência ocorre" (Santos \& Moré, 2011).

Dado o exposto, destacam-se as vivências das crianças e adolescentes em suas famílias, já que é possível que levem para suas vidas adultas os modelos relacionais aprendidos (Colossi, Marasca, \& Falcke, 2015; Mosmann, Zordan, \& Wagner 2011; Pedro, 2013). Deste modo, questiona-se acerca do impacto que a relação dos pais como casal tem na vida dos filhos. Se a disfuncionalidade conjugal contribui para o comprometimento dos relacionamentos amorosos dos filhos na idade adulta, cabe referir que a presença de infidelidade na relação dos pais assume um aspecto relevante neste contexto, já que contribui para a constituição da dinâmica conjugal estabelecida, impactando não só na vida do casal, mas também, da família e dos envolvidos naquele contexto (Falcke \& Wagner, 2005) Diante disso, destaca-se a relevância de investigar de que modo os aspectos concernentes às vivências familiares se expressam em relações pautadas ou não pela infidelidade conjugal. A partir disso, o presente estudo analisou o perfil discriminante de pessoas que cometeram e que não cometeram infidelidade em seus relacionamentos acerca das experiências na família de origem e vivências de violência conjugal. 


\section{Método}

\section{Delineamento}

O presente estudo teve caráter quantitativo, com delineamento descritivo, já que buscou discriminar os grupos que cometeu e não cometeu infidelidade, no que tange à violência conjugal e experiências dos parceiros em suas famílias de origem.

\section{Participantes}

Participaram deste estudo 384 mulheres e 216 homens $(\mathrm{n}=600)$ acessados por meio de mídias sociais. Os participantes tiveram idade igual ou superior a 18 anos, em relações heterossexuais de namoro, casamento ou união estável há pelo menos seis meses. Foram recrutados por conveniência, já que os primeiros foram pessoas das redes sociais das pesquisadoras e foram convidados a indicar outros participantes que se enquadrassem nos critérios de inclusão desta investigação, constituindo uma coleta do tipo "bola de neve".

A amostra se caracterizou segundo os dados apresentados na Tabela 1, a seguir:

TABELA 1

Caracterização da amostra

\begin{tabular}{lcc}
\hline & Frequência & Percentual (\%) \\
Sexo & & \\
$\quad$ Feminino & 384 & 64,0 \\
Masculino & 216 & 36,0 \\
Filhos & & \\
$\quad$ Com filhos & 184 & 30,8 \\
$\quad$ Sem filhos & 414 & 69,2 \\
Status da relação & & \\
Namorando & 215 & 35,8 \\
Morando junto/união estável & 179 & 29,8 \\
Casado oficialmente & 206 & 34,4 \\
Escolaridade & & \\
Sem instrução formal & 1 & 0,2 \\
Ens. Fundamental Completo & 12 & 2,0 \\
Ens. Médio Completo & 219 & 37,0 \\
Ens. Superior Completo & 202 & 34,1 \\
Pós-Graduação Completa & 158 & 26,7 \\
Atividade Remunerada & & \\
Sim & 482 & 80,9 \\
Não & 114 & 19,1 \\
\hline
\end{tabular}

\section{Instrumentos}

Com a finalidade de investigar a infidelidade, violência no casal e as experiências de violência na família de origem, foram utilizados os seguintes instrumentos:
Questionário sociodemográfico: elaborado para o presente estudo, constituiu-se em um questionário, segundo o critério Brasil (ABEP, 2010), para identificação de aspectos sociodemográficos. Os participantes ainda foram questionados acerca de possível comportamento de infidelidade no relacionamento atual, anterior ou ambos.

Family Background Questionnaire (FBQ): elaborado por Melchert (1998a; 1998b; Melchert \& Sayger, 1998) com 179 questões, apresentadas com respostas no formato Likert, de cinco pontos, é composto por 22 subescalas que envolvem aspectos relacionados às recordações do sujeito acerca das experiências vivenciadas em sua família de origem. Este instrumento foi validado para o contexto nacional por Falcke (2003), alcançando um alto índice de confiabilidade de mensuração. Para este estudo foram utilizadas as subescalas de abuso físico paterno e materno, abuso sexual, negligência física, abuso de substância paterno e materno, ajustamento psicológico paterno e materno, aliança parental. O alpha de Cronbach obtido na escala total, para este estudo, foi 0.909 revelando um excelente resultado. Os índices de consistência interna medidos pelo mesmo coeficiente foram analisados em cada subescala utilizada neste estudo e chegaram a resultados entre 0,737 e 0,894 . Foram incluídas, ainda, questões objetivas para investigar infidelidade na família de origem do respondente.

CTS2 (Revised Conflict Tactics Scale): Elaborada por Strauss et al. (1996) e adaptada ao português por Moraes, Hasselmann, e Reichenheim (2002), a escala busca identificar a violência em relações conjugais. Refere-se a um questionário de 78 questões fechadas, que avalia ações do respondente e do seu companheiro(a), de modo recíproco, quanto à negociação, agressão psicológica, violência física, lesão corporal e coerção sexual, formando cinco escalas que representam as respectivas dimensões. Neste estudo, foram utilizadas as dimensões de agressão psicológica, violência física e coerção sexual.

Escala de percepções de infidelidade amorosa (PDIS): proposta por Wilson, Mattingly, Clark, Weidler Bequette (2011), e traduzida para o português para a realização do presente estudo, obedeceu a um rigoroso processo de tradução para o português. É um instrumento do tipo checklist, que busca aferir o nível de concordância do respondente quanto a possíveis comportamentos de infidelidade. O presente definiu os resultados a partir do escore geral e obteve um alpha de Cronbach de 0.846 , confirmando a fidedignidade do instrumento.

Escala de atitudes relacionadas à infidelidade (EAI): proposto por Whatley (2006) é apresentado 
em uma lista de 12 comportamentos associados à infidelidade a fim de que o respondente responda o seu nível de concordância em relação ao referido comportamento. Apresentado como um checklist, e em escala Likert de sete pontos, foi submetida ao mesmo criterioso processo de tradução para o português, por tratar-se de um instrumento elaborado para o contexto norte-americano. No presente estudo, o coeficiente obtido foi 0,796 , referindo a aceitabilidade do instrumento para mensuração a que se propõe (George, \& Mallery, 2003).

Escala de propensão para infidelidade (EPI): Construído e validado por Drigotas, Safstrom e Gentilia (1999), avalia a propensão do sujeito à infidelidade. Igualmente submetida ao criterioso rigor de tradução para o português, esta escala é composta de 11 itens, em uma escala Likert de oito pontos, com vistas a acessar o nível de intimidade emocional, física e cognitiva do respondente com outra pessoa externa ao relacionamento amoroso principal. O objetivo da escala é avaliar os comportamentos de propensão à infidelidade. No presente estudo, o coeficiente obtido foi 0,917 , revelando excelência para mensurar o constructo proposto (George \& Mallery, 2003).

\section{Procedimentos éticos e de coleta dos dados}

Após aprovação no comitê de ética em pesquisa da Universidade (Parecer 13/182), o link de pesquisa foi divulgado e compartilhado nas redes sociais. A partir do interesse e enquadre nos critérios de inclusão, o participante precisava clicar no link da pesquisa e era redirecionado a uma plataforma digital de coleta de dados. Esta investigação está em conformidade com a Resolução 510/2016 do CNS acerca dos cuidados éticos para pesquisas com seres humanos. Deste modo, na primeira página da plataforma, constava um Termo de Consentimento Livre e Esclarecido (TCLE) com a descrição do estudo e informações pertinentes à pesquisa e pesquisadora responsável, em que o participante precisava aceitar, para dar continuidade à coleta de dados. O respondente foi informado, ainda, acerca do anonimato, preservação da identidade bem como a não identificação do IP do computador em que o questionário fora respondido.

\section{Procedimentos de análise dos dados}

Os dados coletados foram examinados quantitativamente, a partir do software SPSS (Statistical Package for the Social Sciences), versão 22, para identificação da prevalência de infidelidade, violência e experiências na família de origem, bem como as possíveis correlações entre variáveis. A partir disso, foi realizada a análise discriminante das dimensões de violência conjugal e das experiências na família de origem, a fim de identificar as variáveis que mais discriminavam os grupos de pessoas que cometem infidelidade e que não cometem.

\section{Apresentação e Discussão dos Resultados}

Inicialmente, foi identificada a prevalência da infidelidade nas relações dos respondentes, conforme apresentado na Tabela 2 e a prevalência das diferentes expressões de violência conjugal, apresentado no Gráfico 1.

TABELA 2

Prevalência de infidelidade referida pelo respondente

\begin{tabular}{lcc}
\hline & Frequência & Porcentagem \\
$\begin{array}{l}\text { Sim, no relacionamento atual } \\
\text { Sim, em um relacionamento } \\
\text { anterior }\end{array}$ & 80 & 13.3 \\
$\begin{array}{l}\text { Sim, em um relacionamento } \\
\text { anterior e no atual }\end{array}$ & 34 & 26.0 \\
Não & 330 & 5.7 \\
Total & 600 & 55.0 \\
\hline
\end{tabular}

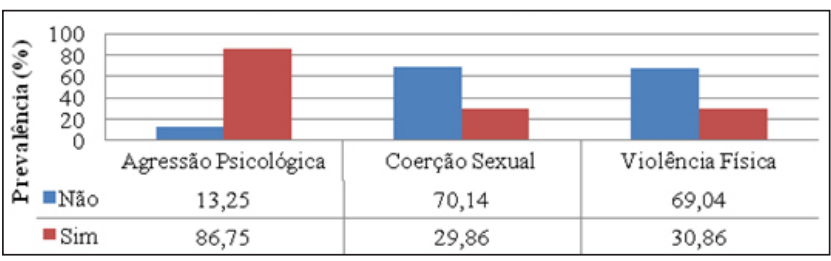

Gráfico 1. Prevalência de violência conjugal

Foram identificadas as correlações entre as referidas dimensões e as experiências do sujeito na família de origem, conforme apresentado na Tabela 3, a fim de mapear as associações existentes.

Foram também identificados os aspectos avaliados pelas escalas utilizadas, a fim de determinar de que forma as variáveis diferenciam os grupos em relação ao cometimento de violência conjugal e experiências nas famílias de origem dos participantes. Deste modo, foi realizada uma análise discriminante entre os grupos.

$\mathrm{O}$ número de funções discriminantes corresponde ao número de grupos menos um (Hair Jr., Anderson, Tatham, \& Black, 2005). A função obtida no perfil discriminante entre os grupos de pessoas que cometeram ou não infidelidade, por ser única, explica $100 \%$ da variabilidade entre os dois grupos. A função discriminante é significativa $\left(\chi^{2}=43,508 ; p<0,001\right)$ e apresenta uma correlação canônica discriminante de 0,437 (Tabela 4). 
TABELA 3

Correlações de infidelidade, violência conjugal e experiências na família de origem

\begin{tabular}{lccc}
\hline & $\begin{array}{c}\text { Atitudes relacionadas à } \\
\text { infidelidade }\end{array}$ & $\begin{array}{c}\text { Percepções } \\
\text { de infidelidade }\end{array}$ & $\begin{array}{c}\text { Propensão para } \\
\text { a infidelidade }\end{array}$ \\
Violência conjugal &, $095^{*}$ &, $104^{*}$ &, 043 \\
$\quad$ Agressão Psicológica &, $105^{*}$ &, 074 &,- 023 \\
Coerção Sexual &, $147^{* *}$ &, 065 &, $092^{*}$ \\
Violência Física & & &, 011 \\
Experiências na famíliade origem &, 016 &, 080 &, 008 \\
Abuso Físico Paterno &, 001 &, 004 &, 076 \\
Abuso Físico Materno &, 081 &, 066 &, 034 \\
Abuso Sexual &, 016 &, $108^{*}$ &, 024 \\
Negligência Física &, 079 &, 018 &, 007 \\
Abuso Substâncias Paterno &, 009 &, 040 \\
Abuso Substâncias Materno &,- 019 &,$- 121^{* *}$ &,- 013 \\
Ajustamento Psicológico Paterno &,- 070 &,$- 105^{*}$ &,- 003 \\
Ajustamento Psicológico Materno &,- 050 &,$- 116^{*}$ & \\
Aliança Parental & & & \\
\hline
\end{tabular}

$* p<0,05 ; * * p<0,01$

TABELA 4

Valores próprios da função obtida

\begin{tabular}{cccccccc}
\hline \multicolumn{10}{c}{ Valores próprios da função obtida } & & \\
Função & Valor próprio & \% de variância & Correlação Canônica & Wilks Lambda & $\chi^{2}$ & $g l$ & $p$ \\
1 & 0,236 & 100,0 & .437 & .809 & 43.508 & 15 & 0,000 \\
\hline
\end{tabular}

Pode-se observar um $\lambda$ de Wilks de 0,809, equivalente a uma variância explicada pela função discriminante de 23,6\%. Com relação à classificação, a função obtida classifica corretamente $64,5 \%$ dos participantes em seus respectivos grupos, sendo um bom índice de classificação preditiva por meio do perfil da função obtida (Tabela 4).

Para a compreensão do que a função está discriminando, utilizaram-se os valores dos centroides. $\mathrm{O}$ grupo de pessoas que nunca cometeram infidelidade apresentou valor centroide de -0,477, enquanto o grupo que já cometeu infidelidade apresentou o valor de 0,490. Esses valores indicam que os grupos estão bem afastados pelo perfil obtido, podendo ser discriminados pelas variáveis apresentadas, sendo que o sinal das correlações obtidas indica a direção favorável a um grupo ou a outro. As variáveis significativas e mais relevantes na capacidade discriminativa entre os grupos estão ordenadas por tamanho absoluto de correlação na matriz estrutural, tendo como ponto de corte 0,07 (Pizzinato \& Sarriera, 2003; Mosmann, Wagner, \& Sarriera, 2008), conforme apresentado na Tabela 5. Do total, as variáveis analisadas e apresentadas na análise discriminante, podem ser divididas em três eixos descritos como: infidelidade, violência conjugal e experiências na família de origem.
TABELA 5

Matriz estrutural das principais variáveis utilizadas na análise discriminante

\begin{tabular}{lc}
\hline Variável & Função l \\
Propensão à Infidelidade & .733 \\
Atitudes de Infidelidade & .640 \\
Negligência Física & .330 \\
Abuso Sexual & .269 \\
Ajustamento Psicológico Materno & -.181 \\
Ajustamento Psicológico Paterno & -.155 \\
Agressão Psicológica & .138 \\
Coerção Sexual & .123 \\
Abuso Físico Materno & .121 \\
Aliança Parental & -.106 \\
Abuso de Substâncias Paterno & .090 \\
Violência Física & .071 \\
\hline
\end{tabular}

As questões do eixo infidelidade que pesaram na discriminância dos grupos foram as que buscam identificar a propensão do sujeito ao envolvimento com alguém fora da relação principal $(0.733)$ e as atitudes de infidelidade (0.640), tornando-os, mais próximos do grupo que cometeu infidelidade. Entretanto, as percepções dos respondentes acerca da infidelidade não 
impactaram na discriminação dos grupos. Sendo assim, a busca de homens e mulheres por maior satisfação sexual, e conjugal de modo amplo, associa-se com o envolvimento dos participantes em relações fora da relação principal (Bozon, 2003; Duarte \& RochaCoutinho, 2011), ao passo que não se diferencia entre os grupos a percepção sobre quais comportamentos se caracterizam ou não como infidelidade.

Com relação ao eixo experiências do sujeito na família de origem que apresentam maior poder discriminante entre os dois grupos, destacam-se a negligência física (0.330), o abuso sexual (0.269), o ajustamento psicológico materno $(-0.181)$ e o ajustamento psicológico paterno (-0.155). No mesmo eixo, com menor poder de discriminação entre os grupos, destaca-se o abuso físico materno (0.121), a aliança parental (-0.106) e abuso de substâncias paterno (0.090). As experiências de negligência física, abuso sexual, abuso físico materno e abuso de substâncias paterno se aproximam, conforme valor dos centroides, do grupo de pessoas que cometeram infidelidade, enquanto que as experiências de ajustamento psicológico paterno e materno, bem como aliança parental, estão na direção do valor de centroide das pessoas que não cometeram infidelidade. Destacam-se, assim, que os resultados corroboram estudos prévios que revelam o impacto de experiências familiares de negligência física (Fang \& Corso, 2007), abuso sexual (Alexander, 2009; Fang \& Corso, 2008) e déficits no ajustamento psicológico materno e paterno (Hughes, Stuart, Gordon, \& Moore, 2007; Lawson \& Rivera, 2008; Murrell, Christoff, \& Henning, 2007; Weisbart et al., 2008; Whiting, Simmons, Havens, Smith, \& Oka, 2009) no envolvimento de contextos conjugais adultos disfuncionais. Chama a atenção que das experiências na família de origem, a que mais discrimina os grupos, na direção do grupo que cometeu infidelidade, é a negligência física que corresponde ao cuidado que o sujeito teve quanto a aspectos básicos referentes à alimentação, vestuário e em situações de doenças ou machucados. A partir disso, pode-se apontar que as experiências de negligência contribuem para a busca de alguém que exerça o cuidado, que manifeste o interesse, que faça um o investimento amoroso em si mesmo. É possível que quando o sujeito não encontre, ou não reconheça isso no parceiro, possa buscar fora da relação principal alguém que possa satisfazê-lo.

Em sentido oposto, as experiências familiares que mais discriminam os grupos e se aproximam daqueles que não cometeram infidelidade correspondem às competências parentais que representam o equilíbrio (ajustamento psicológico materno, paterno e aliança parental) e correspondem respectivamente, à saúde mental das figuras parentais e associação dos pais na educação dos filhos além da ausência de conflitos. Deste modo, cabe destacar que as experiências funcionais do sujeito com as figuras parentais representam aspectos favorecedores da fidelidade relacional.

Já do eixo correspondente à violência conjugal, as questões que compreenderam a agressão psicológica $(0,138)$ e coerção sexual $(0,123)$ revelaram poder discriminatório entre os grupos, estando mais próximas do grupo de pessoas que cometeram infidelidade. Com relação às expressões de violência que mais discriminam os grupos, pesam as agressões psicológicas. Estudos anteriores referem associação das variáveis infidelidade e violência, apontando a primeira como preditora da segunda (Guedes, Silva, \& Fonseca, 2009; Ortiz, Leiva, \& Jacinto, 2009; Wilson, Mattingly, Clark, Weidler, \& Bequette, 2011), mas com foco predominante na associação da infidelidade com a expressão física da violência (Andrade, Mello, \& Dias, 2009). Neste estudo, entretanto, ainda que a violência física tenha se aproximado mais do valor centroide das pessoas que cometeram infidelidade, não apresentou um grande poder de discriminação entre os grupos $(0,071)$. Sendo assim, os resultados alcançados por este estudo apontaram a presença ou não de agressões psicológicas como um aspecto que discriminante dos grupos estudados (.138), se destacando mais do que a expressão física da violência no casal.

\section{Considerações finais}

O presente estudo buscou investigar os aspectos relacionados às expressões da violência conjugal e das experiências na família de origem que se expressam como fatores discriminantes dos grupos que cometeu e não cometeu infidelidade. Os resultados apresentados evidenciaram atitudes e propensão para infidelidade, negligência física, abuso sexual, abuso físico materno, abuso de substância paterno e violência psicológica e sexual as associadas ao perfil de pessoas que já cometeram infidelidade em seus relacionamentos.

Longe de assumir uma compreensão carregada de carga moral, mas buscando compreender a infidelidade como um aspecto de uma relação conjugal, cabe destacar que vivências negativas tanto da infância e adolescência quanto da vida adulta, no que tange ao relacionamento conjugal, se mostram como discriminantes do comportamento de (in)fidelidade. Ora, se o adulto aprendeu como modelo familiar, uma relação marcada pela aliança parental positiva e ajustamento psicológico dos pais, tem maior predisposição às vivências funcionais na vida adulta. Contudo, o que se busca destacar não é 
uma compreensão de certo e errado, mas as experiências consideradas pelos parceiros como positivas ou não. Neste sentido, os resultados apresentados revelaram que as experiências consideradas saudáveis na família de origem mostram-se compatíveis com comportamento fiel na vida adulta. As experiências de ajustamento psicológico materno e paterno além da aliança parental se mostraram associadas ao comportamento fiel, revelando-se como protetoras do envolvimento em contextos de infidelidade.

No sentido inverso, as experiências negativas na infância e adolescência, sejam elas da ordem da violência intrafamiliar ou da ausência de cuidados, se mostram relevantes na predição do envolvimento do sujeito em contextos conjugais com presença de infidelidade, já que as experiências de negligência física, abuso sexual, abuso físico materno e abuso e substâncias paterno foram aspectos que se associaram ao grupo que cometeu infidelidade. Ademais, as três dimensões de violência se mostraram associadas ao grupo que cometeu infidelidade, sendo a agressão psicológica a expressão violenta que mais discriminou os grupos investigados.

Com isso, cabe refletir sobre a relevância de propor intervenções com o casal, seja no que tange às políticas públicas, seja no âmbito privado, no sentido de prevenir e tratar contextos conjugais disfuncionais. Ainda que o presente estudo não tenha tido o objetivo de propor alternativas de intervenção, cabe destacar a possibilidade da terapêutica conjugal, a partir dos aspectos que sustentam a relação, considerando os legados transgeracionais e as características relacionais dos parceiros mostrem-se relevantes no estabelecimento da conjugalidade. Neste sentido, tratar o casal em sofrimento, repercute não apenas na dupla conjugal, mas nos membros da família nuclear e extensa que convivem com a relação.

Este estudo corrobora investigações anteriores, realizadas em diferentes contextos, que referem o peso das vivências familiares na predição de contextos conjugais disfuncionais na vida adulta. Contudo, os resultados não podem ser generalizados dado o tamanho da amostra e o contexto específico em que foi realizado. Ademais, não esgota o estudo da infidelidade e dos aspectos que contemplam o referido fenômeno.

A partir dos resultados desta investigação, destaca-se a relevância de ampliar estudos que associem a expressão psicológica da violência conjugal com aspectos da infidelidade, considerando vivências do sujeito antes mesmo da vida adulta. Ainda que esta investigação possa colocar luz sob esta forma de violência no casal, novos estudos precisam ser realizados a fim de seguir contribuindo com o campo científico ao qual encontra-se vinculado, dada a importância que a ciência tem dado, nos últimos tempos, à violência física, deixando a violência psicológica às margens da maioria das publicações.

\section{Referências}

Alexander, P. C. (2009). Childhood trauma, attachment, and abuse by multiple partners. Psychological Trauma-Theory Research Practice and Policy, 1(1), 78-88. https://doi.org/10.1037/a0015254

Almeida, T. C., Gonçalves, R. A., \& Sani, A. I. (2010). Comportamiento agresivo en el menor: Testimonio de conflictos interparentales. Revista Infancia, Juventud y Ley, 2, 78-81.

Alves, R. A., Pinto, L. M. N., Silveira, E. M., Oliveira, G.L., \& Melo, E. M. (2012). Homens, vítimas e autores de violência: a corrosão do espaço público e a perda da condição humana. Interface - Comunicação, Saúde, Educação, 16(43), 871-883. https://doi.org/10.1590/S1414-32832012005000049

Andrade, M. N., Mello, I. S. B., \& Dias, C. M. S. B. (2009). Sentimentos relacionados a infidelidade matrimonial: um estudo com universitários. Rev enferm UFPE, 3(4), 882-889. https://doi.org/10.5205/reuol.581-3802-1-RV. 0304200912

Baucom, D. H., Snyder, D.K., \& Dixon, Lee J. (2008). Couple Therapy and the Treatment of Affairs. In Gurman, Alan S. Clinical Handbook of Couple Therapy (4a ed.). New York: The Guilford Press.

Bittar, D.B., \& Nakano, A.M.S. (2011). Violência intrafamiliar: análise da história de vida de mães... Texto Contexto Enferm, Florianópolis, 20(1), 17-24. https://doi.org/10.1590/S0104-07072011000100002

Bowker, L. H., Arbitell, M., \& McFerron, J. R. (1990). On the relationship between wife beating and child abuse. In K. Yllö \& M. Bograd (Eds.). Feminist perspectives on wife abuse (pp. 158-174). California: Sage Publications.

Bozon, M. (2003). Sexualidade e conjugalidade: A redefinição das relações de gênero na França. Cadernos Pagu, 20, 131-156. https://doi.org/10.1590/S0104-83332003000100005

Braga, L. L., \& Dell'Aglio, D. D. (2012). Exposição à violência em adolescentes de diferentes contextos: Família e instituição. Estudos de Psicologia, 17(3), 413-320. https://doi.org/10.1590/S1413- 294X2012000300009

Cecconello, A., De Antoni, C., \& Koller, S. (2003). Práticas educativas, estilos parentais e abuso físico no contexto familiar. Psicologia em Estudo, 8, 45-54. https://doi.org/10.1590/S1413-73722003000300007

Cerveny, C. (2011). A família como modelo (2aa ed.). São Paulo, SP: Livro Pleno. 
Coker, A.L., Smith, P.H., McKeown, R.E., \& King, M.J. (2000). Frequency and correlates of intimate partner violence by type: physical, sexual and psychological battering. American Journal of Public Health, 90(4), 553-559. https:// doi.org/10.2105/AJPH.90.4.553

Colossi, P. M., Marasca, A. R., \& Falcke, D. (2015). De Geração em Geração: A Violência Conjugal e as Experiências na Família de Origem. Psico, 46(4), 493-502. https://doi.org/10.15448/1980-8623.2015.4.20979

Costa, C. B. \& Cenci, C. M. B. (2014). A Relação Conjugal Diante da Infidelidade. Pensando Famílias, 18(1), 19-34.

Donovan, S. \& Emmers-Sommer T. (2012). Attachment style and gender as predictors of communicative responses to infidelity. Marriage \& Family Review, 48(2), 125-49. https://doi.org/10.1080/01494929.2011.626670

Duarte, J. P. \& Rocha-Coutinho, M. L. (2011). "Namorido": uma forma contemporânea de conjugalidade? Psicologia Clínica, 23(2), 117-135. https://doi.org/10.1590/S0103-56652011000200008

Dubowitz, H., Black, M. M., Kerr, M. A., Hussey, J. M., Morrel, T. M., Everson, M. D., \& Starr Jr, R. H. (2001). Type and timing of mothers' victimization: effects on mothers and children. Pediatrics, 107(4), 728-735. https://doi.org/10.1542/ peds.107.4.728

Falcke, D. (2003). Águas passadas não movem moinhos: as experiências na família de origem como preditoras da qualidade do relacionamento conjugal. Porto Alegre, RS. Tese de Doutorado. Pontifícia Universidade Católica do Rio Grande do Sul, 185 p.

Falcke, D. (2006). Filho de peixe, peixinho é: a importância das experiências na família de origem. Colóquio, 3(1), 83-97.

Falcke, D. \& Wagner, A. (2007). A dinâmica familiar e o fenômeno da transgeracionalidade: definição de conceitos. In A. Wagner (Org.), Como se perpetua a família? A transmissão dos modelos familiares (pp. 25-46). Porto Alegre: Edipucrs.

Falcke, D., Wagner, A., \& Mosmann, C. P. (2008). The relationship between family-of-origin and marital adjustment for couples in Brazil. Journal of Family Psychotherapy, 19(2), 170-186. https://doi.org/10.1080/08975350801905020

Fang, X., \& Corso, P. S. (2007). Child maltreatment, youth violence, and intimate partner violence. American Journal of Preventive Medicine, 33(4), 281-290. https://doi.org/10.1016/j.amepre.2007.06.003

Fang, X. \& Corso, P. S. (2008). Gender differences in the connections between violence experienced as a child and perpetration of intimate partner violence in young adulthood. Journal of Family Violence, 23(5), 303-313. https://doi. org/10.1007/s10896-008-9152-0

Féres-Carneiro, T. (1998). Casamento contemporâneo: o difícil convívio da individualidade com a conjugalidade. Psicologia Reflexão e Crítica, 11(2), 379-394. https://doi.org/10.1590/S0102-79721998000200014

Féres-Carneiro, T., \& Neto, O. D. (2010). Construção e dissolução da conjugalidade: padrões relacionais. Paidéia, 20(46), 269-278. https://doi.org/10.1590/S0103-863X2010000200014

França, M. (2015). "Quero um amor sem obrigações": notas antropológicas sobre um estudo entre poliamantes. Novos Debates, 2(1): 146-151

Gover, A. R., Park, M., Tomsich, E. A., \& Jennings, W. G. (2011). Dating violence perpetration and victimization among South Korean college students: A focus on gender and childhood maltreatment. Journal of Interpersonal Violence, 26(6), 1232-1263. https://doi.org/10.1177/0886260510368161

Guedes, R. N., Silva, A. T. M. C., \& Fonseca, R. M. G. S. (2009). A violência de gênero e o processo saúde-doença das mulheres. Esc Anna Nery Rev Enferm, 13(3), 625-673. https://doi.org/10.1590/S1414-81452009000300024

Ha, T., Overbeek, G., Vermulst, A. A., \& Engels, R. C. M. E. (2009). Marital quality, parenting, and adolescent internalizing problems: A three-wave longitudinal study. Journal of Family Psychology, 23(2), 263-267. https://doi. org/10.1037/a0015204

Hair Jr., J. F., Anderson, R. E., Tatham, R. L, \& Black, W. C.(2005). Análise multivariada de dados. Porto Alegre: Bookman, 600 p.

Horta, A. L. M. \& Daspett, C. (2010, agosto). A repercussão da traição feminina na dinâmica do casal heterossexual [Resumo]. In Resumos de Comunicações Científicas, IX Congresso Brasileiro de Terapia Familiar (p. 122). Búzios, RJ, ABRATEF.

Hughes, F. M., Stuart, G. L., Gordon, K. C., \& Moore, T. M. (2007). Predicting the use of aggressive confl ict tactics in a sample of women arrested for domestic violence. Journal of Social and Personal Relationships, 24(2), 155-176. https://doi.org/10.1177/0265407507075406

Jablonski, B. (1998). Até que a vida nos separe - A crise do casamento contemporâneo. Rio de Janeiro: Agir. (Originalmente publicado em 1991).

Kerley, K. R., Xu, X. H., Sirisunyaluck, B., \& Alley, J. M. (2010). Exposure to family violence in childhood and intimate partner perpetration or victimization in adulthood: Exploring intergenerational transmission in urban Thailand. Journal of Family Violence, 25(3), 337-347. https://doi.org/10.1007/s10896-009-9295-7

Lawson, D. M. \& Rivera, S. (2008). Male partner abusers' perceptions of family relationship functioning: A comparison of clinically derived abuser types. Journal of Aggression, Maltreatment \& Trauma, 17(1), 59-79. https://doi. org/10.1037/1524-9220.9.2.90

Magalhães, A. S. \& Féres-Carneiro, T. (2007). Transmissão psíquica geracional: Um estudo de caso. In T. Féres Carneiro (Org.), Família e casal: Saúde, trabalho e modos de vinculação (pp. 341-364). São Paulo, SP: Casa do Psicólogo. 
Marasca, A., Colossi, P. M., \& Falcke, D. (2013). Violência conjugal e família de origem: uma revisão sistemática de 2006 a 2011. Temas em Psicologia, 21(1), 221-243. https://doi.org/10.9788/TP2013.1-16

Melchert, T. (1998a). A review of instruments for assenssing family history. Clinical Psychology Review, 18(2), 163-187. https://doi.org/10.1016/S0272-7358(97)00058-5

Melchert, T. (1998b). Testing the validity of an instrument for assessing family of origin history. Journal of Clinical Psychology. Winsconsin, 54(7), 863-875. https://doi.org/10.1002/(sici)1097-4679(199811)54:7<863::aidjclp1>3.0.co;2-g

Melchert, T. \& Sayger, T. (1998). The development of an instrument for measuring memories of family of origin characteristics. Educational and Psychological Measurement, 58(1), 99-118. https://doi.org/10.1177/ 0013164498058001009

Mendonça, L. M. (2009). Infidelidade conjugal: sob a ótica sistêmico-psicodramática. Salvador: Bureau.

Monteiro, C. F. S. \& Souza, I. E. O. 2007. Vivência da violência conjugal: fatos do cotidiano. Psicologia e Sociedade, 16(1), 26-31.

Moré, C. L. O. O. \& Krenkel, S. (2014). (Orgs.). Violência no contexto familiar. [recurso eletrônico]/Universidade Federal de Santa Catarina; Florianópolis: Cartilha do Curso de Atenção a Homens e Mulheres em Situação de Violência por Parceiros Íntimos. Modalidade a Distância, 82 p.

Mosmann, C., Wagner, A., \& Sarriera, J.C. (2008). A qualidade conjugal como preditora dos estilos educativos parentais: o perfil discriminante de casais com filhos adolescentes (Vol. XXII, pp. 161-182). Lisboa: Edições Colibri.

Mosmann, C., Zordan, E., \& Wagner, A. (2011). A qualidade conjugal como fator de proteção do ambiente familiar. In A. Wagner (Eds.). Desafios psicossociais de família contemporânea (pp. 58-71). Porto Alegre: Artmed.

Murrell, A. R., Christoff, K. A., \& Henning, K. R. (2007). Characteristics of domestic violence offenders: Associations with childhood exposure to violence. Journal of Family Violence, 22(7), 523-532. https://doi.org/10.1007/s10896007-9100-4

Ortiz, J. M. C; Leiva, P. G. \& Jacinto, L. G. (2009). Celos y emociones: Factores de la relación de pareja en la reacción ante la infidelidade. Athenea Digital, 15, 39-55.

Pasini, W. (2010). Amores Infiéis: psicologia da traição. Rio de Janeiro: Rocco.

Pedro, M. M. F. (2013). Relação conjugal e relação pais-filhos: estudo de variáveis mediadoras e moderadoras. Tese [Doutorado]. Universidade de Lisboa, Faculdade de Psicologia.

Pilão, A. (2012). Poliamor: um estudo sobre conjugalidade, identidade e gênero Dissertação [Mestrado]. Programa de Pós-Graduação em Sociologia e Antropologia, Universidade Federal do Rio de Janeiro. 128p.

Pinheiro, I. R., Crepaldi, M. A., \& Cruz, R. M. (2012). Entendeu ou quer que eu desenhe? Transições familiares através da visão sistêmica. Fractal: Revista Psicologia [online], 24(1), 175-192. https://doi.org/10.1590/S198402922012000100012

Pizzinato, A. \& Sarriera, J. C. (2003). Competência social infantil: análise discriminante entre crianças imigrantes e não imigrantes no contexto escolar de porto Alegre. Psicologia em Estudo, Maringá, 8(2), 115-122. https://doi org/10.1590/S1413-73722003000200012

Prado, L.C. (2012). As múltiplas faces da infidelidade conjugal. Sinopsys Editora. 158p.

Riggio, H. R. \& Weiser, D. A. (2008). Attitudes toward marriage: Embeddedness and outcomes in personal relationships. Personal Relationships, 15, 123-140. https://doi.org/10.1111/j.1475-6811.2007.00188.x

Rizzon, A.L. C., Mosmann, C. P. \& Wagner, A. (2013).A qualidade conjugal e os elementos do amor: um estudo correlacional. Contextos Clínicos, 6(1), 41-49. https://doi.org/10.4013/ctc.2013.61.05

Rokach, A. \& Philibert-Lignières, G. (2015). Intimacy, Loneliness \& Infidelity. The Open Psychology Journal, 8(2), 71-77. https://doi.org/10.2174/1874350101508010071

Sani, A. I. \& Cunha, D. M. M. (2011). Práticas Educativas Parentais em Mulheres Vítimas e Não Vítimas de Violência Conjugal. Psicologia: Teoria e Pesquisa, 27(4), 429-437. https://doi.org/10.1590/S0102-37722011000400006

Silva, E. C., Rocha, R., Bobato, S. T., Becker, A.P.S. \& Lorenzetti, N. (2015). Heranças Psíquicas Geracionais e Conjugalidade Contemporânea: Uma Revisão Sistemática. Pensando Famílias, 19(1), 19-31.

Scorsolini-Comin, F., Fontaine, A. M. G. V. \& Santos, M. A. (2015). Conjugalidade dos pais: percepções de indivíduos casados e solteiros. Avaliação Psicológica, 14(2), 223-231. https://doi.org/10.15689/ap.2015.1402.07

Snyder, C. R. \& Lopez, S. J. (2009). Psicologia Positiva: uma abordagem científica e prática das qualidades humanas. (R. C. Costa, Trad.). São Paulo: Artmed.

Straus, M. A., Hamby, S. L., Boney-McCoy, S., \& Sugarman, D. B. (1996). The Revised Conflict Tactics Scales (CTS2): Development and Preliminary Psychometric Data. Journal of Family Issues, 17(3), 283-316. https://doi. org/10.1177/019251396017003001

Viegas, T. \& Moreira, J. M. (2010). Concepções da Infidelidade: Um estudo exploratório. Poster apresentado no XII Congresso Iberoamericano de Psicologia. Oviedo, Espanha.

Wagner, A., Predebon, J., \& Falcke, D. (2005). Transgeracionalidade e educação: como se perpetua a família? In A. Wagner (Org.). Como se perpetua a família? A transmissão dos modelos familiares (pp. 93-106). Porto Alegre: EDIPUCRS. 
Weisbart, C. E, Thompson, R., Pelaez-Merrick, M., Kim, J., Wike, T., Briggs, E., \& Dubowitz, H. (2008). Child and adult victimization: Sequelae for female caregivers of high-risk children. Child Maltreatment, 13(3), 235-244. https://doi. org/10.1177/1077559508318392

Whisman, M. A. \& Baucom, D. H. (2012) Intimate Relationships and Psychopathology. Child Family Psychology, 15, 4-13. https://doi.org/10.1007/s10567-011-0107-2

Whiting, J. B., Simmons, L. A., Havens, J. R., Smith, D. B., \& Oka, M. (2009). Intergenerational transmission of violence: The influence of self-appraisals, mental disorders and substance abuse. Journal of Family Violence, 24(8), 639-648. https://doi.org/10.1007/s10896-009-9262-3

Wilson, K., Mattingly, B. A., Clark, E. M., Weidler, D. J., \& Bequette, A. W. (2011). The Gray Area: Exploring Attitudes Toward Infidelity and the Development of the Perceptions of Dating Infidelity Scale. The Journal of Social Psychology, 151(1), 63-86. https://doi.org/10.1080/00224540903366750

Wong, M. S., McElwain, N. L., \& Halberstadt, A. G. (2009). Parent, family, and child characteristics: Associations with mother and father reported emotion socialization practices. Journal of Family Psychology, 23(4), 452-463. https:// doi.org/10.1037/a0015552

Ziviani, C., Féres-Carneiro, T., \& Magalhães, A. S. (2011). Sons and daughters' perception of parents as a couple: Distinguishing characteristics of a measurement model. Psicologia: Reflexão e Crítica, 24(1), 28-39. https://doi. org/10.1590/S0102-79722011000100005

Ziviani, C., Féres-Carneiro, T., \& Magalhães, A. S. (2012). Pai e mãe na conjugalidade: aspectos conceituais e validação de construto. Paidéia (Ribeirão Preto), 22(52), 165-176. https://doi.org/10.1590/S0103-863X2012000200003

Zordan, E. P. (2010). A separação conjugal na contemporaneidade: motivos circunstâncias e contextos. Tese [Doutorado]. Curso de Pós-Graduação em Psicologia, Pontifícia Universidade Católica do Rio Grande do Sul, Porto Alegre.

Dados dos autores:

Patrícia Manozzo Colossi - Doutora, Faculdades Integradas de Taquara - FACCAT e Universidade do Vale do Rio dos Sinos - Unisinos

Denise Falcke - Doutora, Universidade do Vale do Rio dos Sinos - Unisinos.

Endereço para correspondência:

Patrícia Manozzo Colossi

Faculdades Integradas de Taquara - FACCAT

Av. Oscar Martins Rangel, 4500 - Fogão Gaúcho-

95612-150 Taquara, RS, Brasil

<pmcolossi@gmail.com>

Recebido em: 08.01.2017

Aceito em: 16.10.2018 